\title{
A collision-free path-planning method for an articulated mobile robot
}

doi:10.1080/11762320701494434

\author{
P. Quintero-Alvarez ${ }^{1}$, G. Ramirez ${ }^{2}$ and S. Zeghloul ${ }^{2}$ \\ ${ }^{l}$ Instituto Tecnológico de Nuevo León, Departamento de Ingeniería Eléctrica y Electrónica, Av. Eloy Cavazos 2001. \\ Col Tolteca, 67170 Guadalupe, N.L., Mexico \\ ${ }^{2}$ Université de POITIERS, Laboratoire de Mécanique des Solides U.M.R. 6610 C.N.R.S, SP2MI Téléport 2 Bd. \\ Marie et Pierre Curie BP 3017986962 Futuroscope-Chasseneuil CEDEX, France
}

\begin{abstract}
In previous works, we treated the collision-free path-planning problem for a nonholonomic mobile robot in a cluttered environment. We used a method based on a representation of the obstacles on the robot's velocity space. This representation is called Feasible Velocities Polygon (FVP). Every obstacle in the robot's influence zone is represented by a linear constraint on the robot's velocities such that a collision between the robot and the obstacle could be avoided. These constraints define a convex subset in the velocity space, the FVP. Every velocity vector in the FVP ensures a safe motion for the given obstacle configuration. The path-planning problem is solved by an optimization approach between the FVP and a reference velocity to reach the goal. In this paper, we have extended our work to an articulated mobile robot evolving in a cluttered environment. This robot is composed of a differential mobile robot and one or several modules that together form the trailer which are linked by off-center joints. This kind of robot is a strongly constrained system. Even in a free environment, under some circumstances, the robot may be blocked by its trailers in its progression towards the goal. The proposed approach, compared to other methods, has the main advantage of integrating anticollision constraints between the articulated robot itself and the environment, in order to avoid and resolve dead-lock situations. For moving to the final position, the articulated mobile robot uses the FVP and a reference control law, to formulate the constraints method as a problem of minimal distance calculation. This formulation is then solved with the algorithm of minimal distance calculation proposed by Zeghloul (Zeghloul and Rambeaud, 1996). When a dead-locking situation arises and according to the robot-obstacle configuration, we have developed three different modules to solve these conditions. Each module uses a different approach to resolve the blocking situation. In order to show the capabilities of our method to lead the articulated robot to the final position in a stable way, a numerical result is presented.
\end{abstract}

Key words: Nonholonomic systems, collision-free path-planning, feasible velocities polygon, articulated mobile robot, differential mobile robot.

\section{INTRODUCTION}

The path-planning problem for an articulated mobile robot has been the object of numerous researches for many years. Nevertheless, even if some particular solutions for this problem have been proposed, a general solution does not exist.

Corresponding author

P. Quintero-Alvarez

Instituto Tecnológico de Nuevo León

Departamento de Ingeniería Eléctrica y Electrónica, Av. Eloy Cavazos 2001

Col Tolteca, 67170 Guadalupe, N.L., Mexico

Tel.: 52-81-81570511; Fax: 52-81-81570306

Email: pquinter@itnl.edu.mx
Different approaches have been proposed in order to solve the path-planning problem for an articulated mobile robot. Different classes of nonholonomic systems have been identified: kinematic systems in chained form (Tilbury et al. 1993; Murray and Sastry 1993) and in "extended power" form (Kolmanovsky et al. 1996).

Lamiraux et al. (1999) recommend the use of a virtual robot for resolving the instability problem caused when the robot moves backwards. Tilbury et al. propose (1994) an algorithm for obtaining the state feedback law and the change of coordinates to convert the $N$-trailer system into chained form. Canudas de Wit et al. (1997) present a nonlinear feedback control law and a method of path reconstruction for the last trailers for a train-like vehicle used in dangerous environments. Luo and Tsiotras (1998) introduce a method 
for constructing exponentially convergent laws for $n$ dimensional nonholonomic systems in power form.

In all these works, the probable collision between the trailers is not considered. This paper deals with the pathplanning problem between an initial position and a goal position, for an articulated mobile robot, including the collision avoidance between trailers, considered to be mobile obstacles. In this paper, we have revisited our work in (Ramirez and Zeghloul 2001) based on an original and simple result, the Feasible Velocities Polygon (FVP). Now, we apply this constraints method to an articulated mobile robot, composed of a differential mobile robot (the tractor) and several modules (in this article the authors refer to modules as trailers and tows also) linked by eccentric joints (the trailers). In Quintero-Alvarez et al. (2005), we have made an attempt to solve the collision problem between the modules in a free environment: in order to avoid the collision between the robot and the trailer, the FVP is modified to include the collision-free restrictions. The modules in the trailer are treated as moving obstacles.

To obtain the reference velocity needed in our method, we use the control law developed in Ramirez and Zeghloul (2001). It is a nonlinear control law with a very good performance, which makes the robot to converge asymptotically stable to the goal. We use the "virtual robot" concept proposed by Lamiraux et al. (1999) to solve the stability problem when the robot moves backwards.

Like all local path-planning methods, the optimization based on the FVP can lead the robot to deadlock situations. We utilize a heuristic method to solve such situations, based on the application of maneuvers of human conductors. It involves saving the final position and using a sub-goal. First, we utilize a sub-goal that makes the robot moves forward. When the separation angle between the trailers is the maximum, we change the sub-goal in order to make the robot move backward until a position where it can continue the trajectory to the original final position. All these maneuvers are made by applying the same control law.

\section{KINEMATIC MODEL}

The configuration of an articulated mobile robot in a plan can be defined by the following state vector:

$$
\mathbf{q}=\left[x, y, \theta, \varphi_{1}, \varphi_{2}, \ldots, \varphi_{n}\right]^{T},
$$

where $(x, y)$ is the position of a fixed point on the robot, $\theta$ the orientation of tractor, and $\phi_{1}, \phi_{2}, \ldots, \phi_{\mathrm{n}}$ the orientation of the $n$ trailers joined to the tractor, all these orientations are taken with respect to the $X$ axis.

The kinematics model of an articulated mobile robot, for example, the robot shown in Figure 1 (with one module only) can be defined by

$$
\frac{\mathrm{d} \mathbf{q}}{\mathrm{d} t}=f_{1}(\mathbf{q}) v+f_{2}(\mathbf{q}) \omega
$$

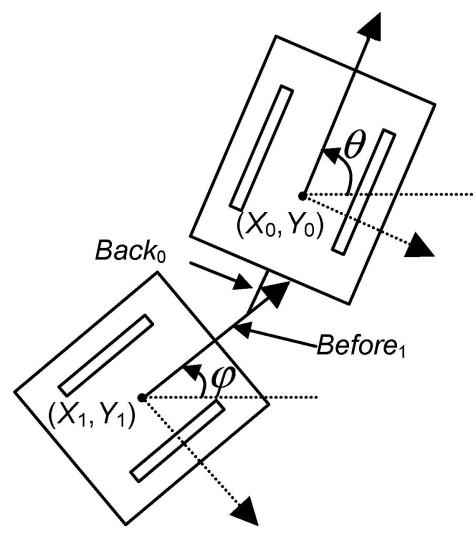

Figure 1 A tractor and a trailer linked for an off-centered joint.

with

$$
\begin{aligned}
& f_{1}(\mathbf{q})=\left[\cos \theta \sin \theta 0-\frac{1}{\mathrm{BEFORE}_{1}} \sin \varphi\right]^{T} \\
& f_{2}(\mathbf{q})=\left[\begin{array}{lll}
0 & 0 & 1
\end{array}\left(-1-\frac{\mathrm{BACK}_{0}}{\mathrm{BEFORE}_{1}} \cos \varphi\right)\right]^{T}
\end{aligned}
$$

where $v$ is the tractor's lineal velocity and $\omega$ is the tractor's angular velocity, $\mathrm{BACK}_{0}$ represents the measurement of the length of the back joint of the robot and BEFORE $E_{1}$ corresponds to the measurement of the length of the front union between the first tow and the tractor.

As a nonholonomic system, this system has nonintegrable velocities restrictions. The nonholonomic restriction for the tractor of this system is given by

$$
\dot{y} \cos \theta-\dot{x} \sin \theta=0 \text {. }
$$

It means that the wheels must roll without slipping.

\section{CONTROL LAW}

In order to obtain a reference trajectory for the robot, we use a control law that assures the global and asymptotical convergence of the system towards the final position. This control law has been developed for a position regulation without final orientation.

From the consideration, we define $\mathbf{q}_{f}=$ $\left[x_{f}, y_{f}, 0, \ldots, 0\right]^{T}$ the desired final position, where the orientation of the tractor and trailer does not matter. The actual robot's position is given by

$$
\mathbf{q}=\left[x, y, \theta, \varphi_{1}, \ldots, \varphi_{n}\right]^{T}
$$

The polar coordinates of the goal, in the robot's frame, $(a, \alpha)$ define the error vector:

$$
z(t)=[a, \alpha]^{T},
$$

where

$a$ is the Cartesian distance from the reference point of the robot to the final position point,

$\alpha$ the angle formed between the $x$ axis of the robot and the final position point. 


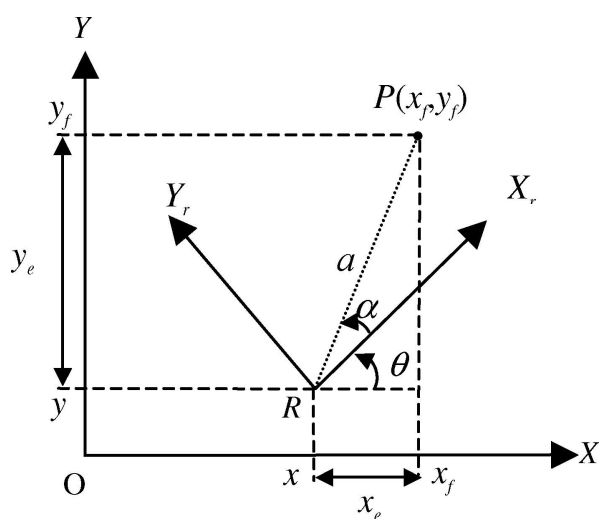

Figure 2 Error variable calculations.

As shown in Figure 2, $(a, \alpha)$ can be obtained from the following expressions:

$$
\begin{aligned}
a & =\sqrt{x_{e}^{2}+y_{e}^{2}}, \\
\alpha & =\tan ^{-1} \frac{y_{e}}{x_{e}}+\theta_{e},
\end{aligned}
$$

where $x_{e}$ represents the difference of the Cartesian distance between the $X$ component of the final position $x_{f}$ and the $X$ component of actual position of the robot, $x ; y_{e}$ represents the difference of the Cartesian distance between the $Y$ component of the final position, $y_{f}$ and the $Y$ component of the real position of the robot, $y$.

From these expressions and the kinematic model of the robot, it is relatively easy to obtain the dynamics of vector $\mathbf{z}$ :

$$
\begin{aligned}
& \dot{a}=-v \cos \alpha, \\
& \dot{\alpha}=\frac{1}{\alpha} v \sin \alpha-\omega .
\end{aligned}
$$

We use the control law proposed by Ramirez and Zeghloul (2001):

$$
\begin{aligned}
v & =k_{1} a \cos \alpha \\
\omega & =\frac{1}{a} v \sin \alpha+k_{2} \alpha
\end{aligned}
$$

to obtain the closed loop system equation:

$$
\begin{aligned}
& \dot{a}=-k_{1} a \cos ^{2} \alpha, \\
& \dot{\alpha}=-k_{2} \alpha,
\end{aligned}
$$

As it has been demonstrated in Ramirez and Zeghloul (2001), using the candidate Lyapunov function:

$$
V(z)=\frac{1}{2}\left(a^{2}+A^{2} \alpha^{2}\right) \geq 0 \quad \forall z,
$$

we have

$$
\dot{V}(z)=-k_{1} a^{2} \cos ^{2} \alpha-k_{2} A^{2} \alpha^{2} .
$$

As $\dot{V}(z)<0 \forall z \neq 0$, then $V(z)$ is a Lyapunov function of the system. The stable convergence to 0 of $(a, \alpha)$ is guaranteed and the robot can reach the final position in a globally and asymptotically stable way.
In conclusion, the control vector proposed allows the robot to reach the final position in a stable and asymptotical way. In addition, as this control law takes into the construction, the kinematics model of the robot, the mobile robot's nonholonomic constraints have been implicitly considered.

\section{ANTI-COLLISION RESTRICTIONS}

The main contribution of this work is that it takes into account the path planning of the geometrical characteristics of the robot to avoid the collision between the trailers and the robot, and the trailers and the obstacles.

In our work, we adapt the constraints method, proposed by Faverion and Tournassaud (1987) as an alternative approach of the potential field method.

The core of the constraint method is the use of a damping constraint, given by the following expression:

$$
\dot{d} \geq-\xi \frac{d-d_{s}}{d_{I}-d_{s}} \quad \text { if } \quad d \leq d_{I}
$$

with $d$ the minimal distance between two objects, $d_{\mathrm{s}}$ the safety distance, $d_{I}$ the influence distance and $\xi$ the positive damping coefficient.

In our previous work (Quintero-Alvarez et al. 2005), we have shown that the constraint imposed by an obstacle over the tractor is given by

$$
(\mathbf{n} \cdot \mathbf{m}) \cdot v_{i}+(\overrightarrow{O P} \times \mathbf{n}) \cdot \mathbf{z} \cdot \omega_{i} \leq \xi \frac{d-d_{s}}{d_{I}-d_{s}}
$$

where $v_{i}$ and $\omega_{i}$ are expressed in function of tractor velocities, $\mathbf{m}$ is the unit vector along the robot $X$ axis and $\mathbf{n}$ is the unit vector along the segment $\vec{P}_{r} \vec{P}_{t}$ of minimal distance between the modules. For a tractor and one trailer as they are shown in Figure 3, we can see that the linear velocity for the tractor and its trailer are the same in point $l$ and we can say that

$$
\begin{aligned}
& A=A_{t}+A_{r}, \\
& B=B_{t}+B_{r},
\end{aligned}
$$

where

$$
\begin{aligned}
& A_{t}=\mathbf{m}_{t} \cdot \mathbf{n}_{t} \quad A_{r}=\mathbf{m}_{r} \cdot \mathbf{n}_{r} \quad \text { and } \\
& B_{t}=\left(\overrightarrow{O P}_{t} \times \mathbf{n}_{t}\right) \cdot \mathbf{z} \quad B_{r}=\left(\overrightarrow{O P}_{r} \times \mathbf{n}_{r}\right) \cdot \mathbf{z}
\end{aligned}
$$

With $\mathbf{m}_{t}$ and $\mathbf{m}_{r}$ the unit vectors of axes $X_{i-1}$ and $X_{i}$ respectively, $\mathbf{z}$ represents the unit vector perpendicular to plan and $\mathbf{n}_{t}$ represents the unit vector across the segment $P_{t}-P_{r}, \mathbf{n}_{r}$ is its negative vector.

We can rewrite the anti-collision restriction for a robot with a trailer as

$$
\begin{gathered}
\left(\mathbf{m}_{t} \cdot \mathbf{n}_{t}+\mathbf{m}_{r} \cdot \mathbf{n}_{r}\right) v+\left[\left(\overrightarrow{O P}_{t} \times \mathbf{n}_{t}\right) \cdot \mathbf{z}\right. \\
\left.+\left(\overrightarrow{O P}_{r} \times \mathbf{n}_{r}\right) \cdot \mathbf{z}\right] \omega \leq \xi \frac{d-d_{s}}{d_{I}-d_{s}}
\end{gathered}
$$




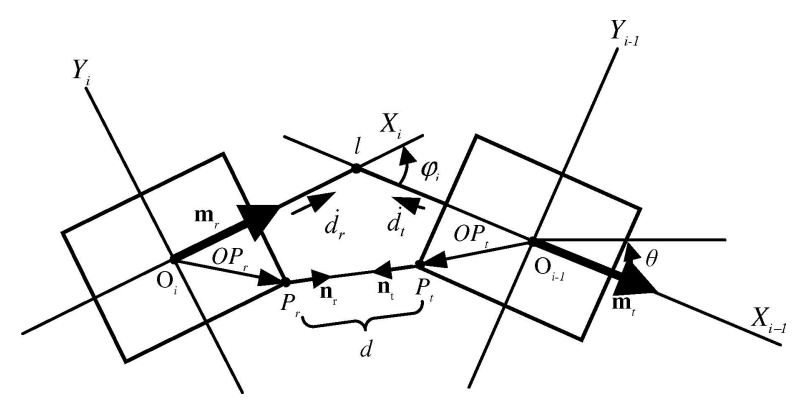

Figure 3 Distance calculation between the tractor and a trailer.

We can conclude that an anti-collision constraint is represented by a linear constraint on the tractor's velocities. The anti-collision constraints between the tractor and the trailers are called internal constraints.

As in Ramirez and Zeghloul (2001), the path-planning problem is resolved as an optimization problem, between the FVP and the reference velocity. This is resolved with the minimal distance calculation algorithm developed by Zeghloul and Rambeaud (1996). The optimization problem can be presented as follows:

$$
\min _{\mathbf{u}} f_{r}=\frac{1}{2}\left\|\mathbf{u}-\mathbf{u}_{\text {goal }}\right\|^{2},
$$

where $\mathbf{u}$ represents the control vector and $\mathbf{u}_{\text {goal }}$ is the reference velocity vector obtained from the control law, obtained by

$$
\mathbf{u}_{\text {goal }}=\left[\begin{array}{l}
k_{1} a \cos \alpha \\
k_{2} \alpha+k_{1} \sin \alpha \cos \alpha
\end{array}\right]
$$

under the non-equality linear constraints

$$
\begin{aligned}
& \left(\mathbf{m}_{t} \cdot \mathbf{n}_{t}+\mathbf{m}_{r} \cdot \mathbf{n}_{r}\right) v+\left[\left(\overrightarrow{O P}_{t} \times \mathbf{n}_{t}\right) \cdot \mathbf{z}\right. \\
& \left.\quad+\left(\overrightarrow{O P_{r}} \times \mathbf{n}_{r}\right) \cdot \mathbf{z}\right] \omega \leq \xi \frac{d-d_{s}}{d_{I}-d_{s}} \\
& |v| \leq V_{\max } \\
& |\omega| \leq W_{\max } .
\end{aligned}
$$

The optimization problem, subject to the internal constraints imposed by the trailers (equation (14)) is a problem of minimal distance calculation between the FVP and the reference point $\mathbf{u}_{\text {goal }}$. The point $\mathbf{u}^{*}$, as we can see in the example of Figure 4 represents the solution to this problem. We use the algorithm developed by Zeghloul and Rambaud (1996) to calculate this minimal distance, and the variation of the configuration vector is then computed using the vector $\mathbf{u}^{*}$.

In addition to the trailers, the obstacles are also represented by linear constraints on the tractor's velocities. These constraints, called external constraints, are obtained by taking into account every trailer and every obstacle in the influence zone of the articulated robot. Figure 4 shows a representation of a FVP (marked area) as a polygon in the tractor's velocity space, obtained after application of

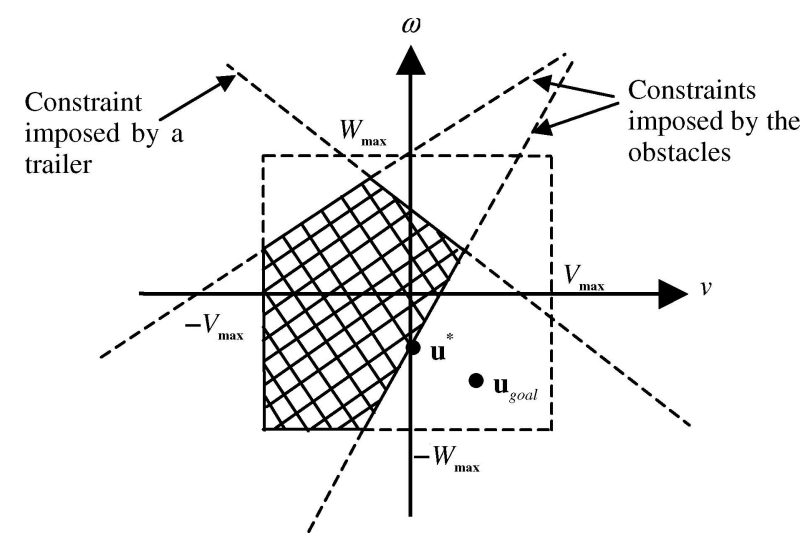

Figure 4 Example of FVP constructions using the constraints imposed by the obstacles over the maximal robot velocities.

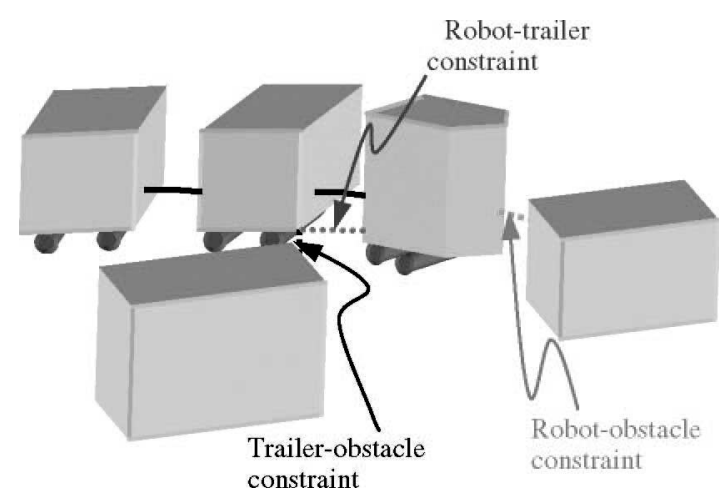

Figure 5 Constraints that can appear in an articulated mobile robot during the path planning in a cluttered environment.

the linear constraints imposed by the modules and the obstacles on the tractor's velocities. This convex polygon represents all the velocities pairs that the robot can use to avoid the collision.

Sometimes the solution to the problem of minimization is found near of $v=0, \omega=0$, producing an oscillation or generating a blocking situation to the robot. When this situation arrives, we must introduce a releasing algorithm for the robot. The method consists of saving the original final position to reach and to give sub-goals, obtained in a heuristic way, in order to make the robot move away of the blocking situation.

Three different kinds of dead-locking situations may appear, in a cluttered environment, as it is shown in Figure 5: dead-lock caused by constraints between trailers, dead-lock caused by constraints between tractor and obstacles and finally dead-lock caused by constraints between trailer and obstacles.

\section{DEAD-LOCKING BETWEEN TRACTOR AND TRAILERS}

The anti-collision constraints between the tractor and the trailers appear in the velocity space of the tractor, when the distance of separation between the tractor and a trailer is lower or equal to the influence distance $d_{I}$. This case has 


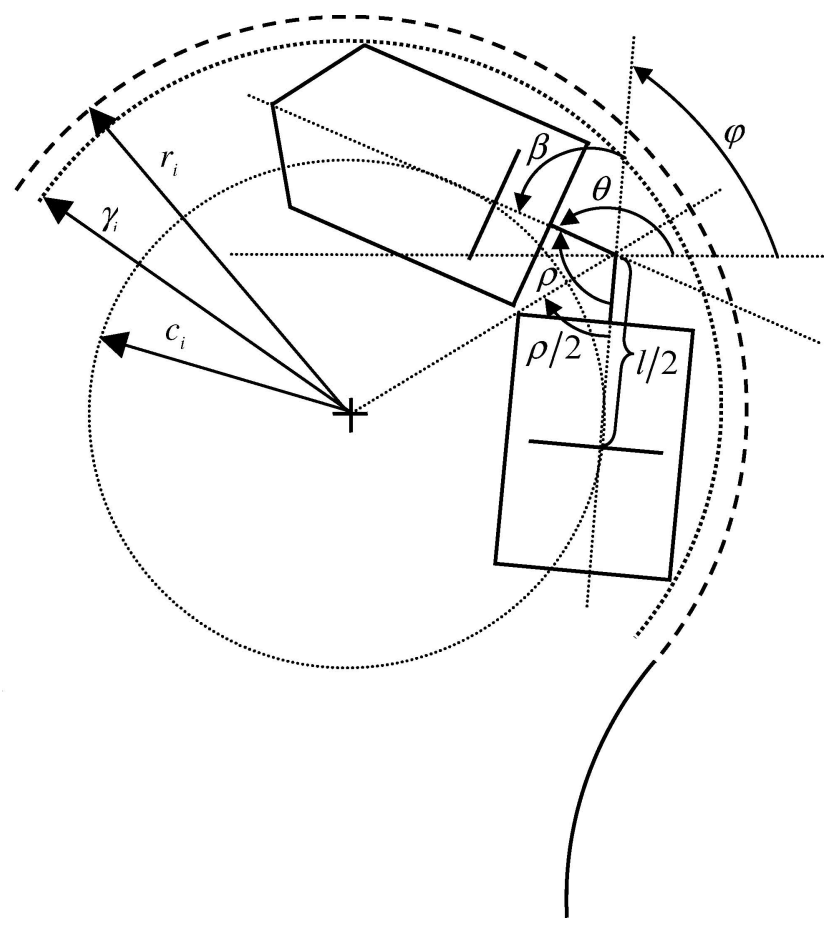

Figure 6 Radio calculation of the curve trajectory followed to circumvent an obstacle $(\theta$ : orientation of the tractor; $\phi$ : orientation of the trailer; $\beta=\theta-\phi$ and $\rho=\pi-\beta ;$ : longitude of the axis of the trailer; $c_{i}$ : radio of the curve in the center of the robot; $\gamma_{i}$ : radio of the curve for circumvents robot; $r_{i}$ : radio of the curve included the safety distance).

been treated in Quintero-Alvarez et al. (2005). No special considerations are made.

\section{DEAD-LOCKING BETWEEN TRACTOR AND OBSTACLES}

In order to avoid a dead-locking between the tractor and the obstacles we will use an influence distance calculated in function of the number of trailers. This consideration can be explained from the fact that the robot needs a larger place to turn around the obstacles depending on the number of modules that it tows.

The influence's distance used by the algorithm must be equal to the radius $r_{i}$, where $i$ stands for the number of modules. The robot must begin to circumvent the obstacle from this distance. Figure 6 shows how this influence distance is obtained.

\section{DEAD-LOCKING BETWEEN TRAILERS AND OBSTACLES}

The anti-collision constraints on the trailers must be mapped to the tractor's velocity space to prevent the collisions. There exist some parameters that must be identified:

1. The tow where the constraint occurs must be identified.

2. The constraints' passage matrix is generated.

3. The constraint produced on the velocity of the trailer must be relocated within the tractor's velocity space.

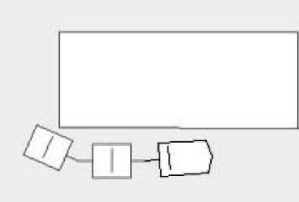

(a)

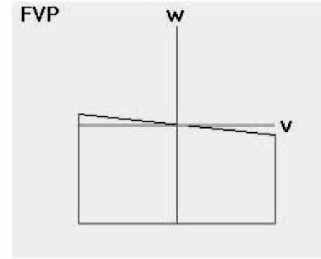

(b)
Figure 7 (a) Situation that generates a constraint on the even trailer, (b) FVP that shows the constraint produced by the obstacle on trailer zero.

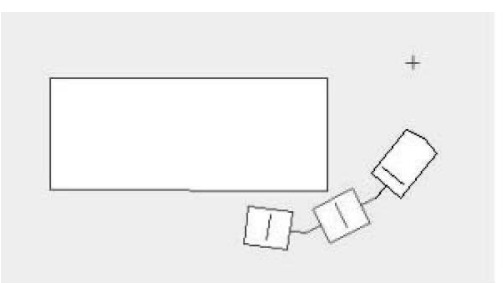

(a)

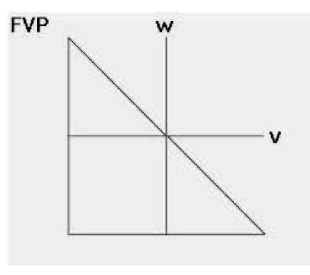

(b)
Figure 8 (a) Situation which generates a constraint on the odd trailer, (b) FVP who shows the constraint produced by the obstacle on trailer 1.

Once the constraint generated on the tow is mapped to the tractor's velocity space, actions must be undertaken to avoid the collision.

Different actions are taken according to the tow the constraint applies.

\section{- Constraint on an even trailer $_{i}$}

Suppose a trailer $_{i}$, where $i=0,2,4,6,8, \ldots$.

When a constraint is produced on an even trailer, as shown in Figure 7, the tractor must take an action that can avoid the tow that continues approaching the obstacle. For this situation, our method proposes to find the projection of the velocity vector under the $X$-coordinate. This action corresponds to preserving the linear component of the velocity and making the angular velocity equals to zero, to prevent that the tractor turns and moves the tow closer to the obstacle. With this action, the tractor will preserve the angular velocity, and the linear velocity used would be then calculated by projection.

- Constraint on an odd trailer $_{i}$

Suppose a trailer $_{i+1}$, where $i=1,3,5,7,9, \ldots$

If a constraint occurred on an odd trailer, as it is shown on Figure 8, the tractor must circumvent the obstacle as if the constraint was produced on itself. That is, because when the tractor circumvents the obstacle, it approaches with the obstacle and the odd trailers will move away from the obstacle. 

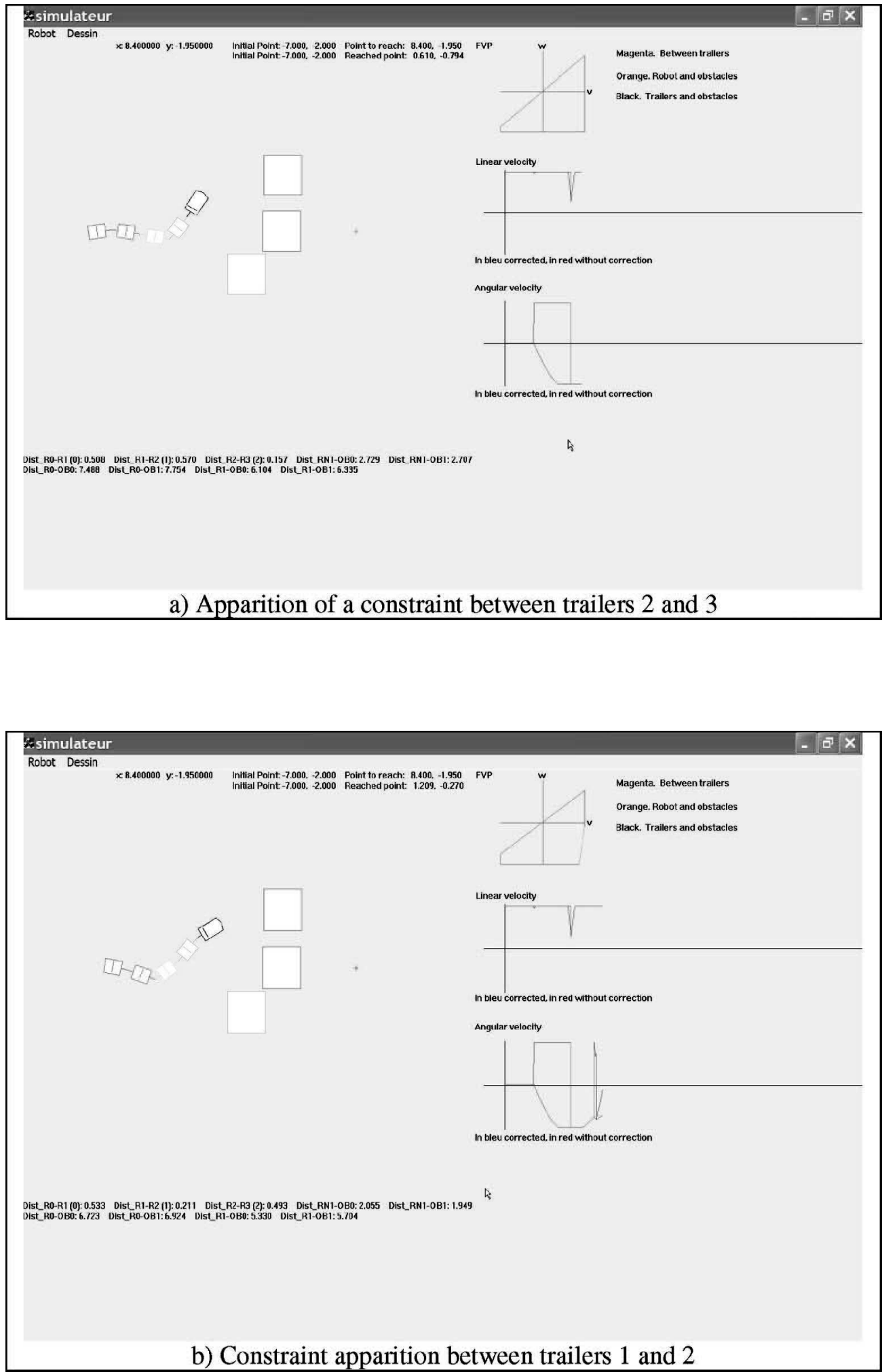

Figure 9 Simulator images showing the apparition of different constraints, due to (a) apparition of a constraint between trailers 2 and 3, (b) constraint apparition between trailers 1 and 2, (c) apparition of a constraint between trailers 0 and 1 and between the robot and the obstacle, (d) apparition of a constraint between the robot and an obstacle, (e) the robot contouring the obstacle which genera a constraint over the robot, (f) the robot contouring the obstacle with the apparition of a constraint between a trailer and the obstacle, $(\mathrm{g})$ constraint apparition between trailer 2 and the obstacle, $(\mathrm{h})$ constraint apparition between trailer 1 and the obstacle, and (i) image showing the entire path the robot has developed to leave the constraints. 


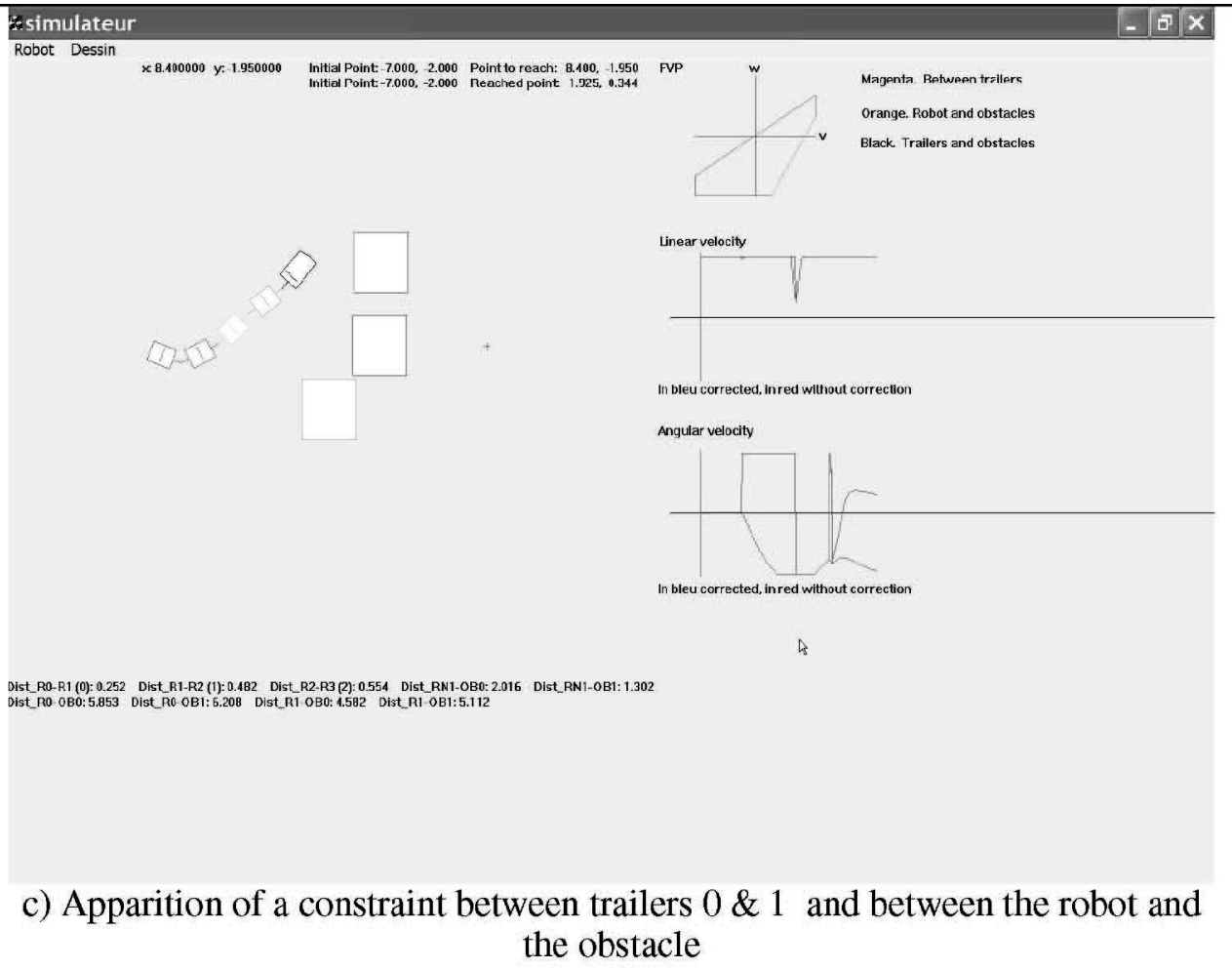
the obstacle

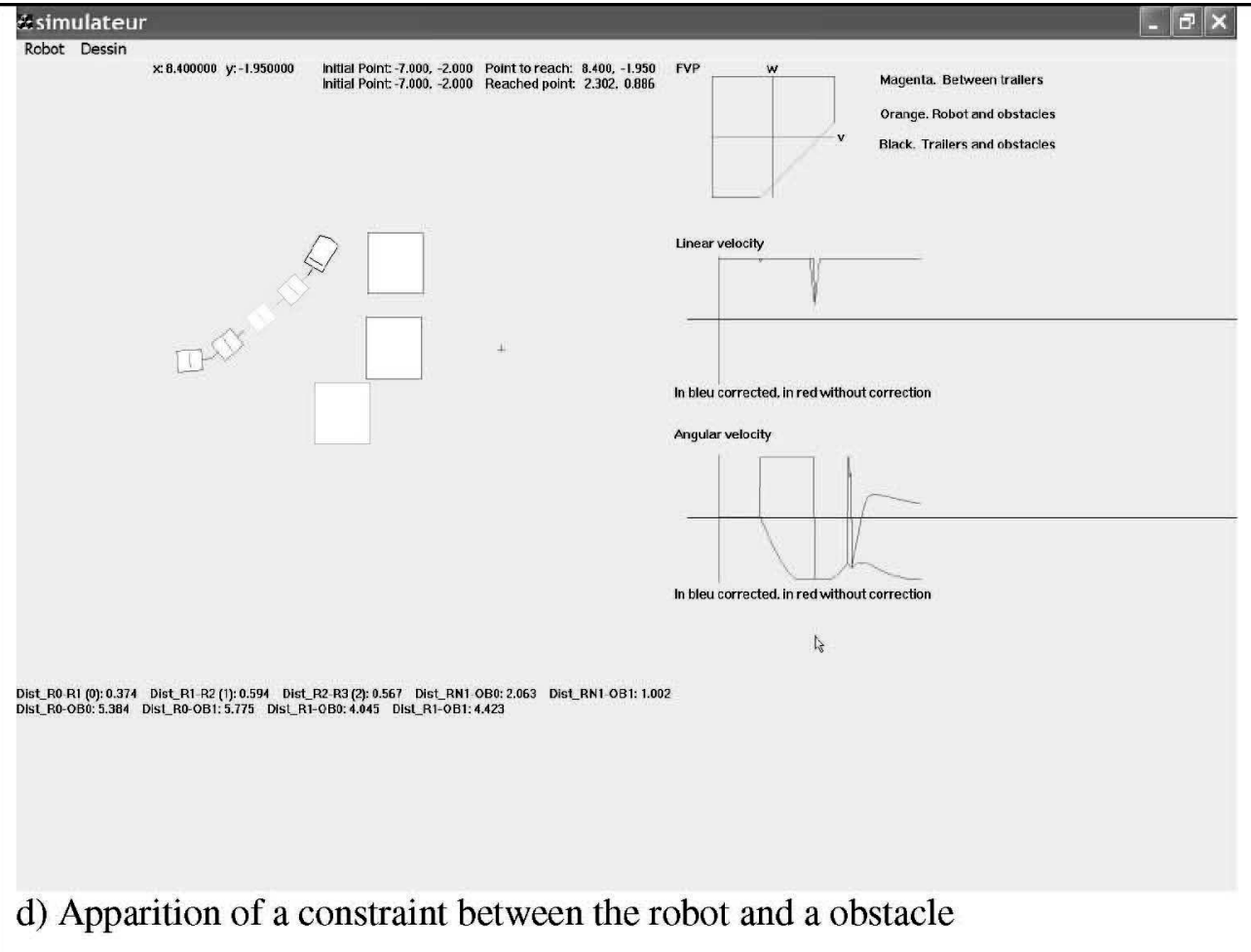

Figure 9 Continued 

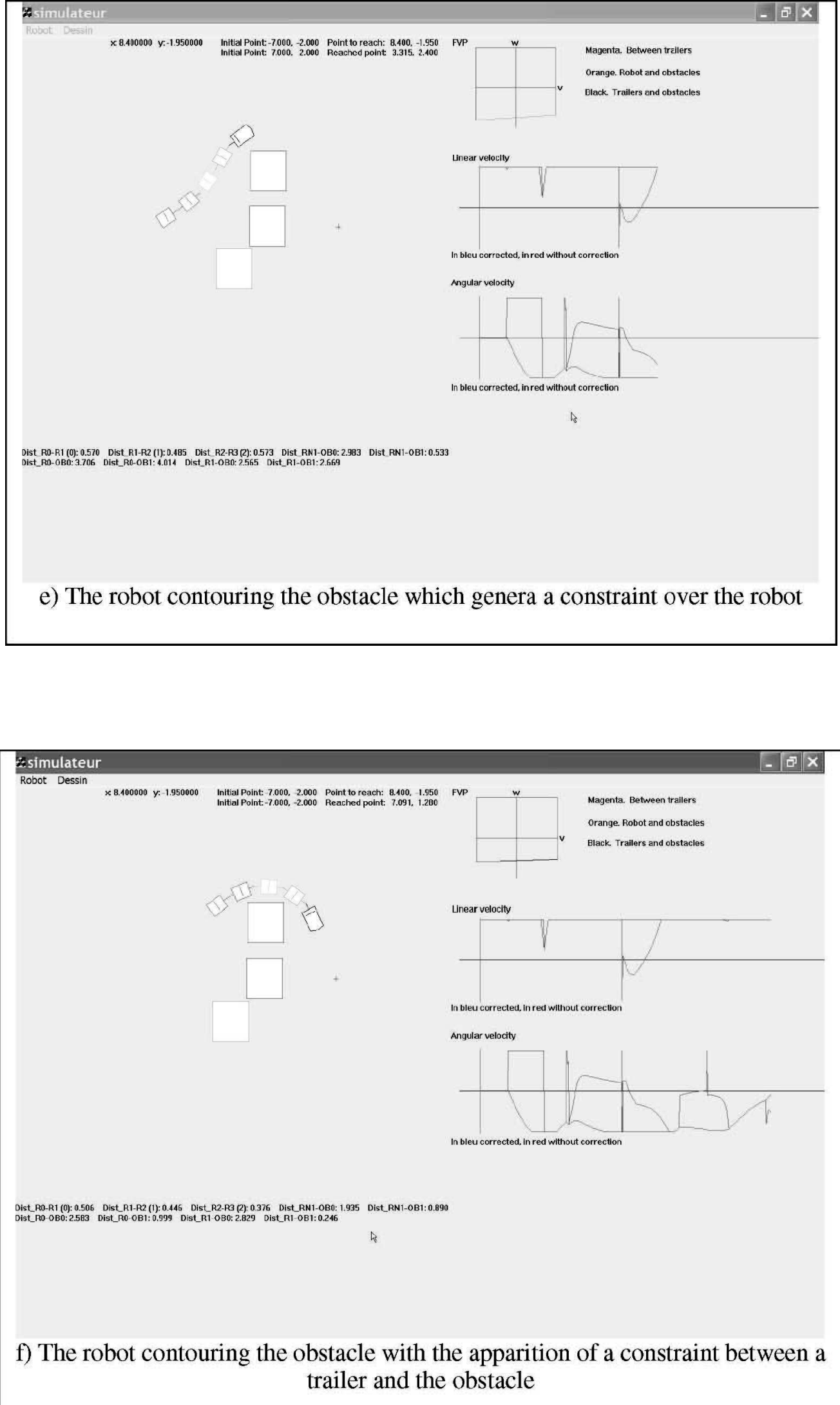

Figure 9 Continued 


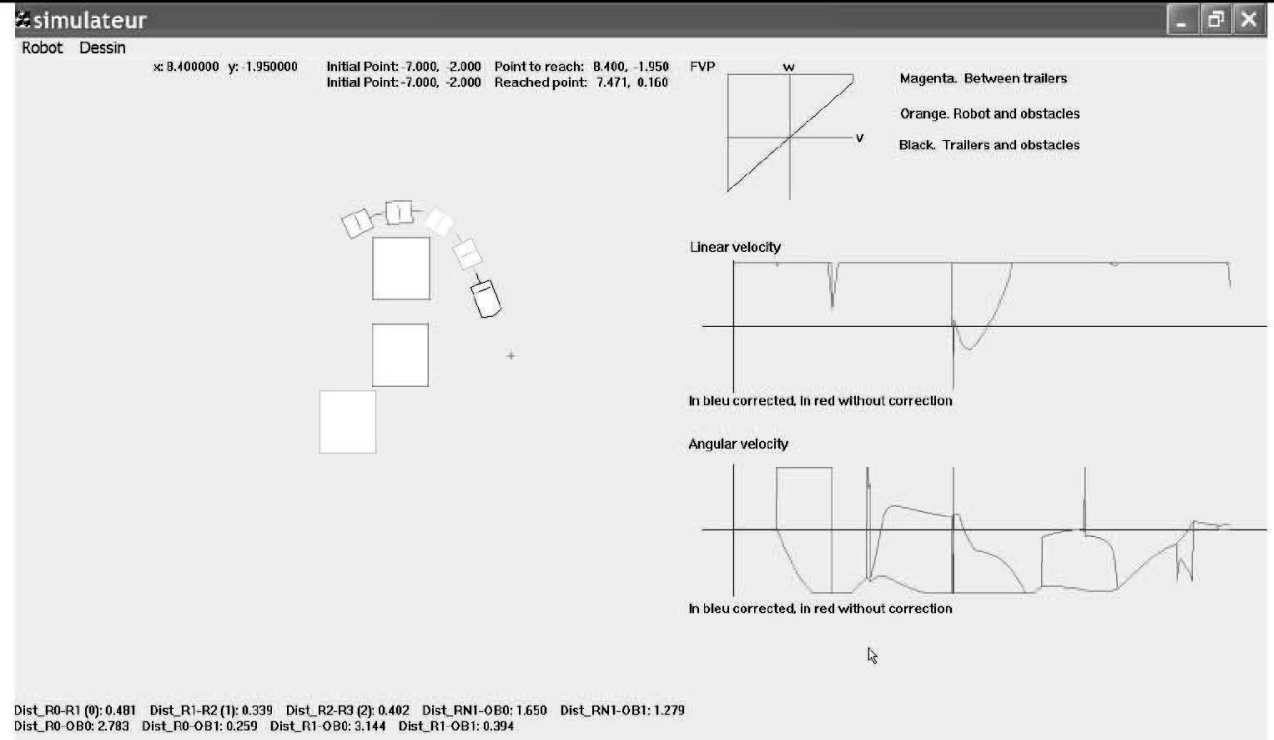

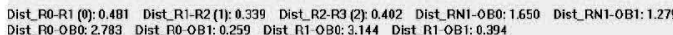

g) Constraint apparition between trailer 2 and the obstacle

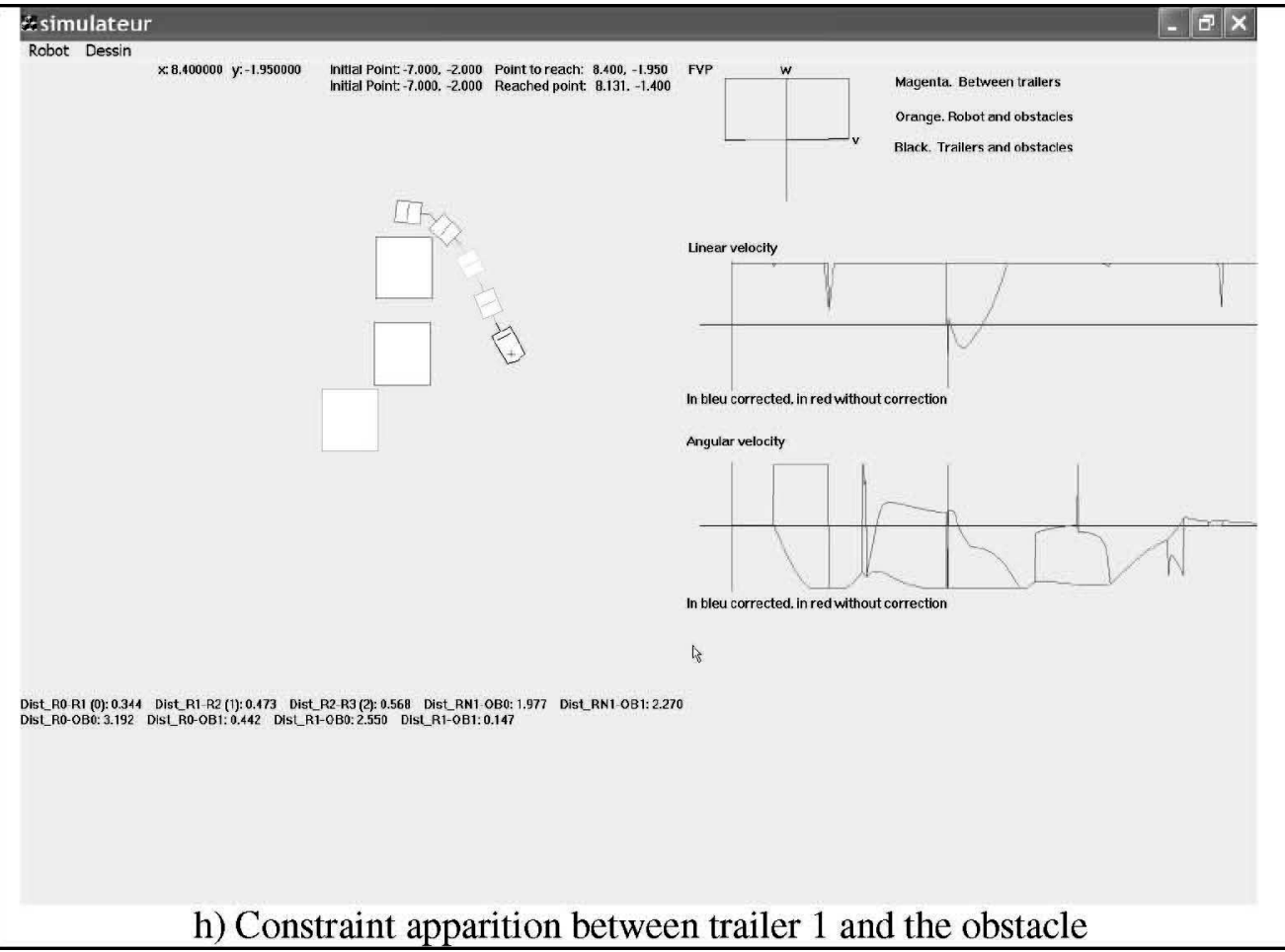

Figure 9 Continued 


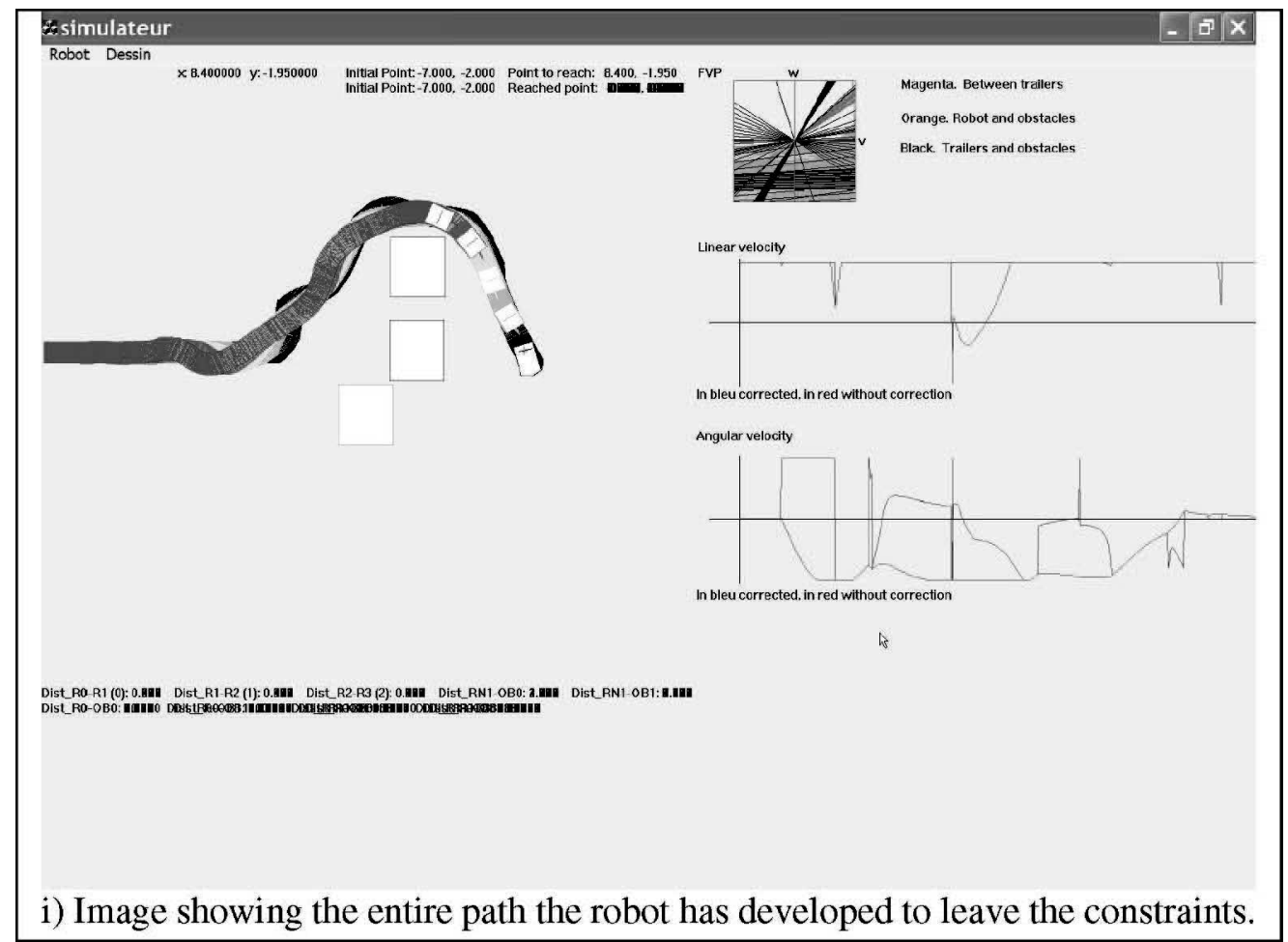

Figure 9 Continued

\section{Numerical example and FVP construction}

In this example, we show the path planning for an articulated mobile robot composed by the tractor and four trailers, from the initial position $(-7,-2,0,0,0,0,0)$ and the final position $(10.125,0.625,0,0,0,0,0)$. The cluttered environment is unknown. The coefficients $k_{1}$ and $k_{2}$ for the reference velocity calculation are both equal to 0.7 , the velocity limits are $v_{\max }= \pm 1.0 \mathrm{~m} / \mathrm{s}$ and $\omega_{\max }=$ $\pm 1.0 \mathrm{rad} / \mathrm{s}$, and

$$
\begin{gathered}
\xi=1.0, \\
d_{s}=0.1, \\
d_{I}=0.3 .
\end{gathered}
$$

Figure 9 shows the articulated mobile robot's progression. These images were taken at the instant the constraints arise. In (a) it is shown a constraint between the first and second trailers and the first module is activated. In the same image, the corresponding PVA is shown. In (b), two constraints are present simultaneously; the first one appears between the second and third trailers and the second one is generated between the tractor and an obstacle. Because the robot is composed of four trailers, the second constraint appears even if the obstacle is relatively away, in order to give more space to manoeuvring. In (c) we observe the evolution of this constraint. In the image (d) the robot is contouring the obstacle. In (e) the robot moves towards the goal, and a constraint between the tractor and the obstacle appears; and in (f) the robot contours the obstacle and a constraint occurred between the first tow and the obstacle. In ( $\mathrm{g}$ ) and $(\mathrm{h})$, the constraints appear between the second and third tow and the obstacle, respectively. And finally in (i) the trajectory carried out by the robot is shown.

\section{CONCLUSION}

In this paper we have extended our path-planning method based in the FVP for an articulated robot in a cluttered environment.

As in our previous works we use the control law that has been proven as an efficient one. This method treats the modules of the trailer as moving obstacles, allowing the robot to approach the final position while avoiding collision between the tows and the robot, between the robot and the obstacles and between the tows and the obstacles. This is made following a stable trajectory of reference, which takes into account the system's nonholonomics constraints.

The constraints imposed by the trailers are mapped as linear constraints on the tractor's velocity space to form the feasible velocities polygon. The free collision trajectory is calculated by a minimization problem between the robot's actual position and the reference trajectory. If a dead-locking situation is detected, our method recognizes it and uses three different algorithms according to the robot's configuration when this situation is generated. This method is well adapted for real-time applications using very short computing times. 


\section{REFERENCES}

Canudas de Wit C, Ndoudi-Likoho AD, Micaelli A. 1997. Nonlinear control for a train-like vehicle. Int 7 Robotics Res, 16(3): 300-319.

Faverjon B, Tournassoud P. 1987. A local based approach for path planning of manipulators with high number of degrees of freedom. In IEEE Proc. International Conference on Robotics and Automation, pp. 1152-1159.

Ramirez G, Zeghloul S. 2001. Collision-free path planning for nonholonomic mobile robots using a new obstacle representation in the velocity space. Int 7 Robotica, 19 : $543-555$.

Kolmanovsky I, Reyhanoglu M, McClamroch NH. 1996. Switched mode feedback control laws for nonholonomic systems in extended power forms. Sys. Control Lett, 27:29-36.

Lamiraux F, Sekhavat S Laumond JP. 1999. Motion planning and control for Hilare pulling a trailer. IEEE Trans Robotics Automat, 15(4): 640-651.

Luo J, Tsiotras P. 1998. Exponentially convergent control laws for nonholonomic systems in power form. Syst Control Lett, 35 : 87-95.

Murray RM, Sastry SS. 1993. Nonholonomic motion planning: steering using sinusoids. IEEE Trans Automat Control, 38 : $700-716$.
Quintero-Alvarez P, Ramirez G, Zeghloul S. 2005. A collision-free path planning method for an articulated mobile robot in a free environement, In Proceedings of IDETC/CIE 2005, Long Beach, CA., Vol. 2.

Sordalën OJ, Canudas de Wit C. 1992. Exponential control law for a mobile robot: extension to path following. Proceedings of IEEE International Conference on Robotics and Automation, pp 2158-2163.

Spong MW, Vidyasagar M. 1989. Robot Dynamics and Control, New York: Wiley.

Tilbury D, Murray R, Sastry S. 1994. Trajectory generation for the N-trailer problem using Goursat normal form, U.C. Berkeley ERL Memorandum, No. M93/12.

Tilbury D, Sordalen O, Bushnell L, Sastry S. 1993. A multi-steering trailer system: conversion into chained form using dynamic feedback, U. C. Berkeley ERL Memorandum No. M93/55.

Walsh G, Tilbury D, Sastry S, Murray R, Laumond JP. 1994. Stabilization of trajectories for systems with nonholonomic constraints, IEEE Trans Automat Control, 39:216222.

Zeghloul S, Rambeaud P. 1996. A fast algorithm for distance calculation between convex objects using the optimization approach, Robotica, 14:335-363. 

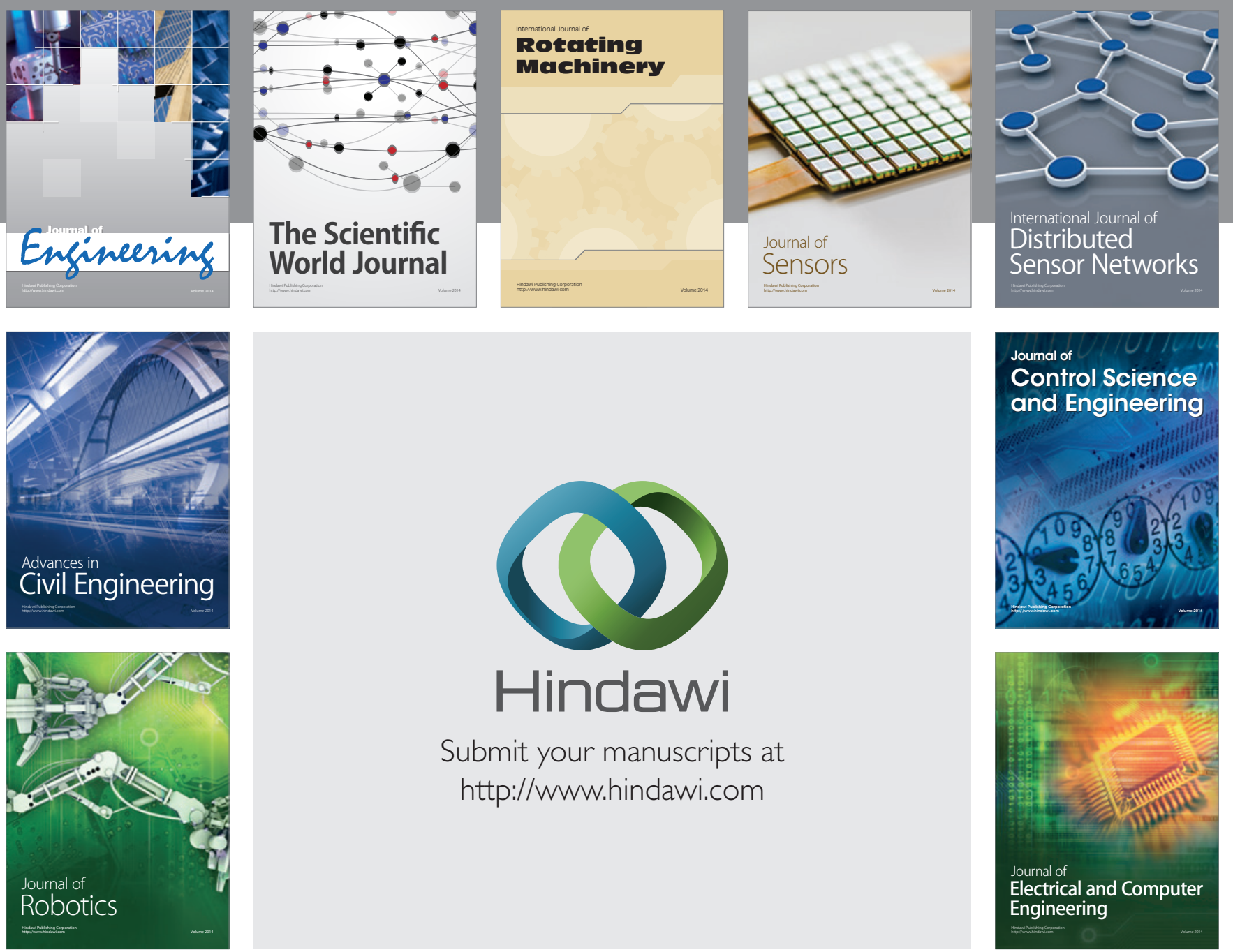

Submit your manuscripts at

http://www.hindawi.com
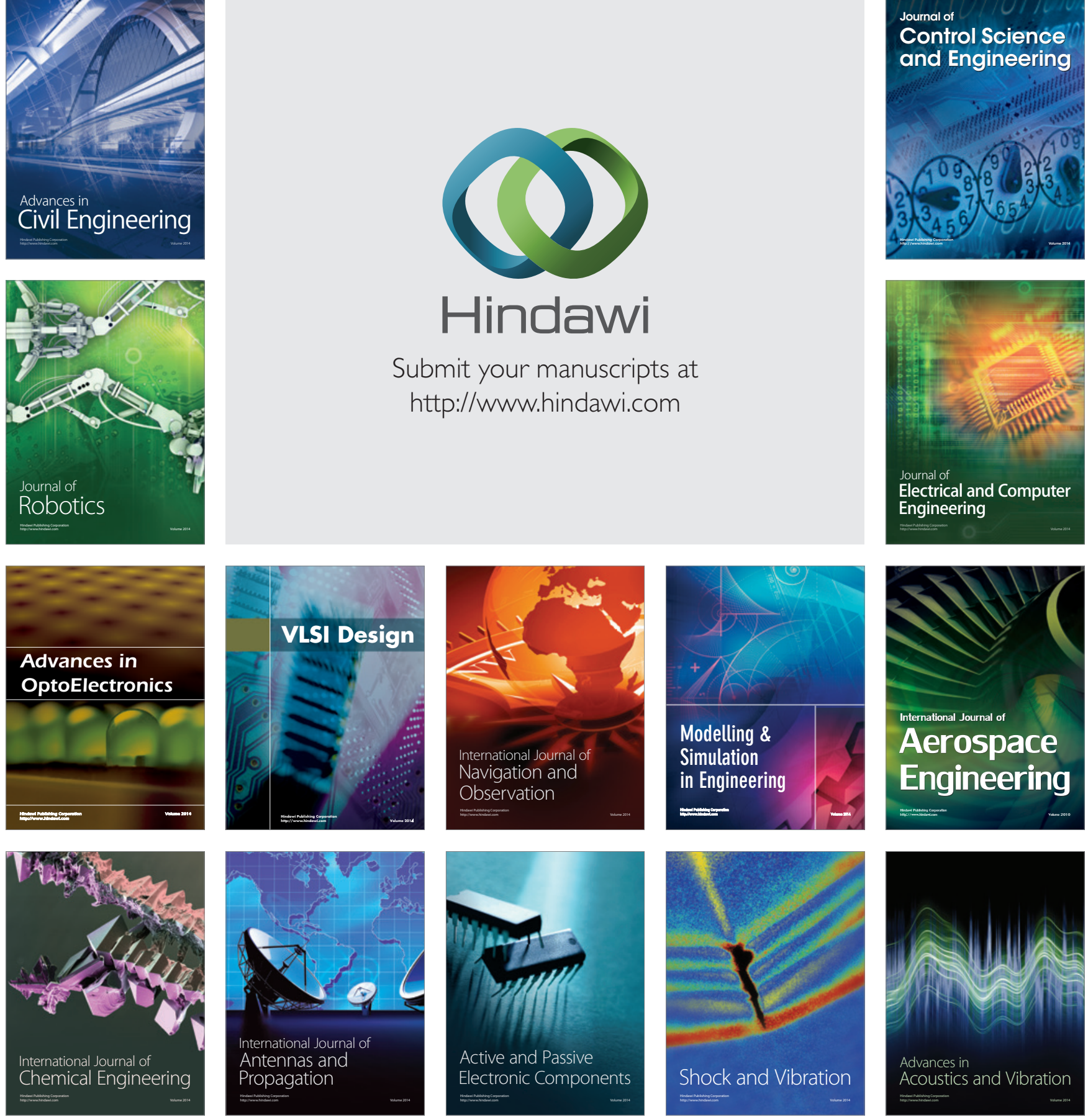